[Berliner entomologische Zeitschrift XVIII. 1874.]

\title{
Ueber die genealogischen Systeme Haeckels, besonders die sog. Gastraeatheorie.
}

\section{$\mathrm{S}_{\mathrm{e}}$} naturae den Weg zur leichteren Uebersicht und Erkennung der zahlreichen Thierformen gegeben hat, sehen wir alle bedeutenderen Zoologen, sei es direkt, sei es indirekt, auf ein Ziel hinstreben: auf das natürliche System. Bald auf diesem bald auf jenem Wege suchte man dasselbe zu erreichen, aber wenn man auch, wie ja nicht ausbleiben konnte, erhebliche Verbesserungen erlangte, so schien doch das Ziel immer in weitere Ferne gerückt.

Man verzweifelte endlich, und während ein Theil der Forscher zur Lösung der Aufgabe die Kenntnifs möglichst aller Thierspecies forderte, leugnete der andere überhaupt die Realität eines natürlichen Systems; man begnügte sich endlich mit derjenigen Anordnung, welche dem Ideale am nächsten kam und in der That den bedeutendsten Fortschritt der Zoologie seit Linné bezeichnete: mit der Typenlehre Cuvier's. Denn während man nach Linné sich das System als eine ununterbrochene Stufenleiter vom unvollkommensten bis zum vollkommensten Organismus vorstellte, wies Cuvier so bedeutende anatomische Verschiedenheiten im Bau der Thiere nach, dafs man genöthigt wurde nicht eine, sondern mehrere solche Stufenleitern anzunehmen, deren einzelne Formen zusammen je einen Typus bildeten. Man unterschied deren vier: Wirbelthiere, Weichthiere, Gliederthiere, Radiärthiere.

Das Cuvier'sche System bildete die Basis aller neueren Systeme, und wenn auch im Einzelnen vieles geändert wurde, so blieb doch die Ansicht bestehen, dafs man es wirklich im Thierreiche mit verschiedenen streng gesonderten Bauplänen zu thun habe, deren Zahl man jedoch allmählich von vier auf sieben erhöhte. Zwar hatten schon zu Cuvier's Zeiten einzelne Männer die Existenz ei- 
nes einheitlichen Bauplanes der Thiere aufgestellt und im Gegensatz zu Linné's Satz: „species tot sunt diversae, quot diversas formas ab initio creavit infinitumens" die Variabilität und Abstammung der Arten von einander gelehrt, doch vermochten sie diese Ansicht Cuvier's Lehre gegenüber nicht hinreichend zu stützen, und ibre Lehre fiel der Vergessenheit anheim. Darwin's unsterbliches Verdienst ist es die Lehre von der Uniformität des Bauplanes neu geschaffen und durch sein umfassendes Wissen fest begründet zu haben. Seine Werke "Entstehung der Arten", "Variiren der Thiere und Pflanzen im Zustande der Domestikation", "Abstammung des Menschen" bezeichnen eine neue Epoche unserer Wissenschaft.

Die Descendenz-Theorie hat in so kurzer Frist die Zustimmung aller denkenden-Forscher erlangt, und greift so sehr in alle wichtigeren Fragen sowohl der Wissenschaft als des Lebens ein, dafs es wohl kaum einen Gebildeten, sicher aber keinen Zoologen geben wird, der sie nicht zum Gegenstand seines Studiums gemacht hat. Es würde daber an diesem Orte überflüssig sein auf eine Auslegung derselben einzugehen, vielmehr möge die Hervorhebung des einen werthvollen Ergebnisses genügen, des Satzes: die Arten sind nicht geschaffen, sondern baben sich aus anderen Arten durch Vererbung und Anpassung entwickelt.

Die durch die Darwin'sche Theorie bedingte Umwandlung unserer Ansichten über das System setzte Haeckel in seiner Gener. Morph. auseinander. Es erscheint bei ihm als ein „übersichtlich nach der Blutsverwandtschaft geordnetes, durch kompakte morphologische Charakteristiken motivirtes Sach- und Namensregister der Organismen".

Das natürliche System kann nur ein genealogisches sein. In dem Studium der Entwickelung der Arten, die unsere jetzige Lebewelt bilden aus solchen, die früheren Perioden angehörten, liegt der Weg zur Erreichung dieses Zieles. Da nun aber die Palaeontologie nur einen verschwindend geringen Bruchtheil der erforderlichen Vergleichsobjekte zu liefern im Stande ist, und auch diese in meist sehr unbefriedigendem Erhaltungszustand, so müssen andere Momente zur Lösung der Verwandtschaftsfragen der Organismen herbeigezogen werden.

Das wichtigste derselben, ja fast das einzige ist nun die Embryologie. Das Studium der Entwickelungsgeschichte des Individuums, die Ontogenie (Haeckel), welcher erst durch C. F. Wolff, Baer, Bischoff, Rathke, Remak u. A. im Anfang dieses Jahrhunderts eine würdige Stellung in der Zoologie geschaffen worden ist, 
wirft ein unerwartetes Licht auf die Entwickelungsgeschichte der Art. Ich kann hier natürlich nicht näher auf die Parallele der Ontogenie und Phylogenie (Entwickelungsgeschichte der Art und des Stammes [Haeckel]) eingehen, doch möge man mir gestatten in gedrängter Kürze ein Beispiel zu erwähnen.

Die unbefruchteten Eier aller vielzelligen Thiere unterscheiden sich nur unwesentlich von einander, auch die ersten Veränderungen des befruchteten Eies zeigen im Grofsen und Ganzen eine auffallende Uebereinstimmung. Erst im weiteren Verlaufe der Entwicklungsgeschichte treten Erscheinungen auf, welche uns ermöglichen die grölsere Abtheilung des Thierreichs zu erkennen, welcher ein gerade beobachteter Keim angehört. Aber eine ganze Reihe von Entwicklungsvorgängen mufs erst abgelaufen sein, ehe wir im Stande sind in demselben z. B. ein Wirbelthier zu erkennen.

In dieser Weise geht es fort, und noch an ziemlich weit entwickelten Embryonen ist es unmöglich zu entscheiden, ob wir es mit einem Reptil, einem Vogel oder einem Säugethier zu thun haben.

Noch in der achten Wocbe ist der Embryo des Menschen kaum von einem Hundeembryo zu unterscheiden, und die Uebereinstimmung desselben mit dem Affen, und nun gar mit einem der $A n-$ thropomorpha wird noch viel längere Zeit andauern.

Alle Wirbelthiere zeigen in einem gewissen frühen Stadium der Entwicklung Kiemenspalten, welche nur bei den Fischen persistiren, bei den übrigen Vertebraten rückgebildet werden, und wie diese, so sind ibnen eine grofse Zahl von Embryonalorganen gemeinsam, die in niederen Klassen zu bleibenden werden, in höheren spurlos verschwinden.

Das Erscheinen solcher in niederen Gruppen persistenter Organe an den Embryonen höherer Thiere mufs nun als Vererbung erklärt werden, und man kann z. B. demnach behaupten, dafs sämmtliche Wirbelthiere sich aus ausgestorbenen Arten entwickelt haben, welche Kiemenspalten und mehrere Paare von Aortenbögen trugen. Wir kommen demnach zu dem Schlufs, dafs, wie Haeckel es geistvoll ausdrückt, die Ontogenie eine abgekürzte Rekapitulation der Phylogenie ist, eine Behauptung, deren Berechtigung durch eine grofse Zahl von Thatsachen allem Zweifel entzogen wird.

Auf diesem Satz fufsend kann der Zoologe hoffen, seine mühevollen Anstrengungen zur Auffindung eines natürlichen Systems vom Erfolge gekrönt zu sehen, und die Resultate, die schon jetzt auf diesem Wege erreicht sind, berechtigen zu den schönsten Hoffnungen. Fritz Müller setzte durch seine Studien über die Entwick- 
lung der Crustaceen in geistvollster Weise die Verwandtschaftsverhältnisse dieser Gruppe auseinander, und Haeckel publicirte in seiner oft genannten Generellen Morphologie Bd. II. den ersten Versuch eines genealogischen Systems des Pflanzen-, Protisten- und Thierreiches.

Wir lassen das Pflanzen- und Protistenreich, unter welch letzteres Haeckel aufser den Moneren, Amoeben, Diatomeen, Flagellaten, Myxomyceten, Noctilucen und Rhizopoden auch noch die Spongien stellt, aufser Betracht, um uns seinem System des Thierreiches zuzuwenden. Er unterscheidet hier abweichend von den allgemein angenommenen sieben Typen 5 Stämme oder Phylen, denn er zieht Würmer und Arthropoden als Articulata zusammen, während die Protozoen zu den Protisten entfallen und die Infusorien auffallenderweise noch zu den Würmern gestellt werden. Während er den Irrthum Burmeister's zurückweist, welcher allein dem Speciesbegriff Realität zuspricht, dieselbe jedoch für die gröfseren Abtheilungen leugnet, sieht er allein in dem Phylum eine reale Kategorie, basirend auf der Blutsverwandtschaft aller dazu gehörigen Formen. Ein Phylum umgreift "die Summe aller Organismen, welche von einer und derselben einfachsten, spontan entstandenen Stammform ibren gemeinschaftlichen Ursprung ableiten ".

Jeden dieser 5 Phylen leitet er daher von einem autogonen (durch Urzeugung entstandenen) Moner ab, ein allerdings sehr gewagter Versuch, da ja doch schon damals, wenn auch Kowalewsky's und Kupfer's Arbeiten über Ascidien und Amphioxus noch nicht erschienen waren, eine genügende Zahl von Formen bekannt waren, welche als Bindeglieder bald dem einen, bald dem anderen Typus zugetheilt wurden und die Haltlosigkeit dieser Annahmen gerade nach Haeckel's Princip darlegten. Allerdings leugnet er auch nicht die Berechtigung, welche die Annahme einer gemeinsamen Urform für das ganze Thierreich hätte.

In seiner "Natürlichen Schöpfungsgeschichte" (d̊ritte Auflage 1872 ) hat er dieses System in mancher Beziehung verändert. Vornehmlich durch Wiederaufnahme des Phylum der Würmer, das ihm jetzt eine hohe Bedeutung erlangt. Hier nimmt Haeckel selbst eine einzige Stammform des gesammten Thierreiches an. Diese Urform, ein autogones Moner, habe die Planula (daher Planulata), eine bewimperte, aus gleichartigen Zellen zusammengesetzte.Thierform hervorgehen lassen, welche nun einerseits den Wurmstamm, andererseits den Zoophyten (Coelenteraten)-Stamm, zu welchem er nun auch richtig die Spongien als älteste Gruppe stellt, entstehen 
liefs. Da er nun aber aus den Würmern auch die anderen 4 Phylen ableitet, so werden diese äufserst ungleichwerthig, denn dem Zoophyten-Stamm stehen alle anderen 5 Phylen gegenüber.

Haeckel tritt uns also hier mit einer wichtigen Modifikation seines ersten Versuches entgegen, und wenn man genau zusieht, giebt er damit seine frühere Definition des Phylum auf. So lange er für ein jedes derselben die Abstammung von einem autogonen Moner behauptet, sind dieselben allerdings der Ausdruck einer Blutsverwandtschaft, sie sind eine reale Kategorie. Jetzt sind sie das nicht mehr, denn da er einen monophyletischen Ursprung des ganzen Thierreiches annimmt, sind auch die Phylen unter sich blutsverwandt, und es verliert dadurch natürlich das Phylum die Bedeutung einer realen Kategorie. Höchstens könute man mit Milderung der strengen Definition die Zoophyta als eine solche den übrigen 5 Typen gegenüberstellen. Trotzdem behält Haeckel aber die Bezeichnung selbst bei. Die Phylen-Lehre, welche ja doch im Grunde nichts anderes als die nach Maafsgabe der Descendenztheorie umgemodelte Typenlehre ist, wäre demnach aufgegeben.

Jetzt aber ist nur noch ein kleiner Schritt zu thun, und wir gelangen zu der Gastraeatheorie, welche als das Resultat der eingehenden Untersuchungen Haeckel's über die Schwämme und die Infusorien zu betrachten ist. Die Gesichtspunkte, auf denen dies neue genealogische System gegründet ist, sind allerdings wesentlich andere. Sie beruhen vor allem auf der Annahme einer wahren Homologie der zwei primären Keimblätter bei allen Thieren, mit Ausnahme der Protozoen, welche letztere nur den Werth einer Zelle haben.

In der Entwicklungsgeschichte der meisten, namentlich niederen Thiere, tritt uns der Embryo oder die Larve, bald nachdem die Furchungserscheinungen abgelaufen sind, in der Form einer zweischichtigen Blase entgegen, deren Innenraum durch eine Oeffnung mit der Aufsenwelt kommunizirt. Man bezeichnete diese Larven, deren Oberfläche meistens Wimpern trägt, in Gemeinschaft mit ähnlichen Formen früher als Planula (T. I. F. 9). Haeckel giebt ihnen in präciserer Abgrenzung den Namen Gastrula. In dem fast allgemeinen Auftreten dieser Form sieht er eine so grofse Bedeutung, dafs er annehmen zu dürfen glaubt, sie sei die Stammform aller mehrzelligen Thiere, und sie nun als eine hypothetische längst ausgestorbene Art mit dem Namen Gastraea bezeichnet. Die beiden Schichten der Blase fafst er als die beiden primären Keimblätter auf, die äufsere als das Haut- oder Dermalblatt, die innere als das 
Darmblatt (Darmdrüsenblatt). Nach ibm ist das mittlere Blatt, welches bei den meisten Thieren eine so gewaltige Massenhaftigkeit erlangt, das Muskelblatt erst aus jenen beiden entstanden, daher als eine sekundäre Bildung aufzufassen. Die Spaltung desselben liefert dann das Körpermuskelblatt einerseits, das Darmmuskelblatt andrerseits, während der durch dieselbe entstandene hohle Raum die Leibeshöhle repräsentirt, deren Bildung erst das Auftreten eines Blutgefäfssystems ermöglicht. Dieser Umstand scheint ihm von tiefgreifender Wichtigkeit, und er scheidet daher die Thiere, welche nach seiner Auffassung eine echte Leibeshöble und daher auch ein Gefäfssystem besitzen, als Coelomati oder Haemataria (Blutthiere) von den Acoelomi oder Anaemaria (blutlose Thiere). Da er nun die innere Zellenschicht der Gastrula, aus welcher sich das Entoderm, d. i. die Auskleidung des Gastrovascularraums der Coeleuteraten entwickelt, als Darmdrüsenblatt ansieht, so spricht er diesen eine Leibeshöhle ab; mit anderen Worten, er nimmt diesen Hohlraum, den man in Uebereinstimmung mit Leuckart allgemein als Leibeshöhle ansieht, als Darm in Anspruch.

Sein genealogisches System ist nun folgendes: die Protozoën, zu denen die Moneren, Amoeben, Gregarinen, Infusorien gebören, werden wieder von dem Protistenreich getrennt, um die Urgruppe einzelliger Thiere darzustellen, aus welcher sich die mehrzelligen monophyletisch entwickelt haben.

Speciell sind es die Amoeben, welche er als primäre Stammform ansieht; aus ihr sollen sich einerseits die Gregarinen und Infusorien, welche letztere also abweichend von der früheren Anschauung als einzellige Thiere aufgefafst werden, andrerseits durch eine hypothetische Morula-Form (T.I. F. 7) (als Morula bezeichnet man das Ei, nachdem es durch oft wiederholte Klüftung eine Maulbeerform angenommen hat) bindurch die ebenfalls hypothetische Gastraea entwickelt haben. Diese letztere repräsentirt die niedrigste Form der Darmthiere oder Metazoen, die, wie oben hervorgehoben, durch die zwei primären Keimblätter charakterisirt werden. Aus der Gastraea sollen sich nun wiederum zwei Stämme entwickelt haben, beide noch obne Leibeshöble und Blut: die Zoophyten (Coelenteraten) und die Acoelomen-Würmer, und zwar soll sich bei den ersteren durch Anpassung an die festsitzende Lebensweise der radiale Bau, bei den letzteren durch Anpassung an frei kriechende, und doch wohl auch schwimmende Bewegung der bilateral-symmetrische ausgebildet haben. Endlich hätten sich aus den blutlosen Würmern die Würmer mit Leibeshöhle und Blut hervorgebildet, aus denen 
zuletzt, getrennten Ursprungs, die Echinodermen ', Arthropoden, Mollusken und Vertebraten hervorgegangen wären.

Während daher die Principien der Anordnung erheblich andere geworden sind, ist das System selbst keiner gleich bedeutsamen Aenderung unterlegen. Wie früher bieten die Würmer, deren Phylum Haeckel jetzt in die zwei wohl kaum haltbaren Phyla der Blutlosen und Blutführenden spaltet, und zwar speciell die letzteren, die Stammgruppe der vier höheren Phyla, während $z$ wischen Planulaten und Zoophyten die hypothetische Gastraea tritt.

Die erheblichsten Aenderungen zeigt noch die Ordnungseintheilung der Würmer, doch auch sie erscheinen nicht wichtig genug, namentlich in ihrer hypothetischen Form um diejenige systematische Bedeutung, welche der Autor selbst seiner Theorie beilegt, zu rechtfertigen. Namentlich scheint uns die Gastraea nicht mehr nöthig gewesen zu sein, um die Typenlehre Cuvier's und Baer's zu stürzen. Man darf behaupten, dafs seit der allgemeinen Anerkennung der Descendenz-Theorie von allen bedeutenden Zoologen die Typen-Theorie stillschweigend als beseitigt angesehen worden ist. Hierfür sprechen zahlreiche Stellen der neueren Literatur. Wir wollen nur an Carus' Geschichte der Zoologie, Claus' Grundzüge, ferner aber an Gegenbauer, Haeckel's eigene Schriften und Ray Lancaster's jüngste Publikation erinnern. Zwar hat sich der Ausdruck „Typus" erbalten, doch nur als Bezeichnung für einen gröfseren Kreis von einzelnen Formen, welche durch Blutsverwandtschaft mit einander zusammenhängen. Das Wort ist von der Bedeutung eines Bauplanes herabgesunken zu der eines ebenso abstrakten Begriffes, wie die übrigen Kategorien des Systemes. Es wäre in Ansehung dessen vielleicht besser es ganz zu verbannen.

Aber ist es denn etwas anderes mit dem Phylum? Auch dieser terminus technicus hat selbst im Munde Haeckel's unbemerkt eine andere Bedeutung angenommen, wie wir oben zu zeigen in der Lage waren. Der Ausdruck, sich damals eng an die Umgrenzung des Typus anschliefsend, pafst nach seiner ursprünglichen Definition nicht auf die jetzigen Phyla Haeckel's, sondern er begreift diese alle in sich.

Da wir diese Zeilen nur als eine referirende Darstellung betrachten, so kann es sich hier nicht um eine Besprechung des pro

1) Haeckel fafst nämlich mit Gegenbauer und Anderen die Stachelhäuter, also Seesterne, Seeigel u. s. w. als Thierstöcke, nicht aber als einzelne Thiere auf. 
und contra handeln. Doch würde man uns mit Recht der Einseitigkeit beschuldigen, wenn wir die Gegner dieser ebenso kühnen als geistreichen Theorie unberücksichtigt liefsen, deren Einwendungen nicht zu unterschätzen sind.

$\mathrm{Daf}$ es an solchen nicht fehlen würde, war zu erwarten, doch sei uns gestattet hier nur die wichtigsten derselben, ausführlicher begründet durch Claus (die Typenlehre und Haeckel's sog. GastraeaTheorie. Wien 1874), zu erwähnen. Die Einwürfe dieses bekannten trefflichen Zoologen richten sich gegen den Grundstein des Gebäudes: gegen die Homologie der Keimblätter und der ursprünglichen Darmanlage. Claus weist vor Allem darauf bin, dafs die zweischichtige Gastrula, wie zahlreiche Beobachtungen lebren, auf (4) verschiedene Weisen aus der einschichtigen Keimblase (T.I. F. 8) entstehen könne, und dafs das anders entstandene Entoderm demnach nicht ohne Weiteres in allen Fällen homologisirt werden dürfe. Entsteht dasselbe durch Einstülpung, so ist es zusammengesetzt aus Zellen der primären einschichtigen Keimblase selbst, entsteht es jedoch durch peripherische Theilung des einschichtigen Zellenlagers in eine äufsere und eine innere Lage, so sind die Zellen des Entoderms Derivate derjenigen des Exoderms, ein Unterschied von so fundamentaler Bedeutung, dafs man sich mit der Bemerkung Haeckel's, es werde ja schliefslich dadurch dasselbe Resultat erreicht, unmöglich beruhigen kann. Aber auch die weitere Entwickelung der Keimblätter, besonders die des mittleren, des Mesoderms, und seiner beiden Schichten giebt weitere wichtige Anhaltspunkte zur Bekämpfung der Haeckel'schen Lehre.

Ueber die Bildungsgeschichte des Mesoderms herrschen so grundverschiedene, durch Beobachtungen unterstützte Ansichten, dafs man absolut nicht im Stande ist dieselben auf einen einheitlichen Vorgang zu beziehen. Die Arbeiten Kowalewsky's selbst, auf die sich Haeckel besonders beruft, führen uns mehr als ein Beispiel so durchgreifender Verschiedenheiten vor, dafs die Homologisirung der einzelnen Blätter im Haeckel'schen Sinne die $\mathrm{Zu}$ verlässigkeit der Beobachtungen des ersterwähnten ausgezeichneten Forschers in Abrede stellen heifst. Auch die Beobachtungen anderer Embryologen über die Bildung des Mesoderms bieten uns ähnliche Differenzen, und wenn sich auch ein Theil derselben, da sie sich auf dasselbe Thier beziehen, als ungenau wird erweisen müssen, so berechtigt uns doch jedenfalls der jetzige Stand der Frage nicht zu der festen Aufstellung des Satzes, dafs beide primäre Keimblätter, also sowohl Exoderm als Entoderm, sich an der 
Bildung des Mesoderms betheiligen; eine Annahme, die nur durch eine einzige Beobachtung Kowalewsky's an Amphioxus, deren Richtigkeit aber vom Autor selbst als fraglich bezeichnet wird, gestützt werden könnte.

Sehen wir so das Fundament der Theorie nicht ohne Erfolg und Berechtigung angegriffen, so erleidet auch der specielle Aufbau des Systems, das übrigens im Grunde mit dem von Gegenbauer in seiner vergleichenden Anatomie 1870 aufgestellten identisch ist, eine Opposition, die sich namentlich gegen die von jenem berühmten Anatomen abweichende Eintheilung der Würmer in solche mit und solche ohne Leibeshöhle und Blut richtet.

Die verschiedene Auffassung der beiden primären Keimblätter bedingt im Grunde schon selbst eine Meinungsdifferenz in Betreff der Leibeshöhle, die nun noch dadurch verschärft wird, dafs Claus als Leibeshöhle die primäre Höhle im Innern der einschichtigen Keimblase ansieht, die bei der Einstülpung zu einem Exoderm und Entoderm trennenden Spalt wird, während Haeckel unter Leibeshöhle den sekundären Spalt versteht, welcher durch das Auseinanderweichen der beiden Platten des mittleren Blattes hervorgeht: Bildungen, die vollkommen von einander verschieden sind, namentlich wenn man wie Haeckel eine vorherige Verwachsung dieser beiden Blätter annimmt.

Es würde mich zu weit führen noch näher auf Einzelheiten einzugehen, auch glaube ich das mehr auf ein Specialfeld gerichtete Interesse der Leser dieser Blätter nicht zu lange in Anspruch nehmen zu dürfen. Ich will daher mit der Bemerkung schliefsen, dafs, so sehr man auch für jetzt noch die Richtigkeit der Haeckel'schen Theorie zu bestreiten, auch die daraus gezogenen Schlüsse zurückzuweisen berechtigt sein mag, man doch auf der andern Seite den geistreichen Gedankengang und die geniale Darstellung dieses vielangefochtenen Forschers und Philosophen zu bewundern gezwungen ist, dessen anregenden Arbeiten ein guter Theil der Fortschritte der neueren Zoologie zuzumessen ist, dem jedoch ein weniger schroffer Ton seinen Fachgenossen gegenüber zu wünschen wäre.

Dr. W. H. Rolph in Leipzig.

Erklärung der beigegebenen Abbildungen auf Taf. I.

Fig. 7. Maulbeerstadium des gefurchten Eies. Morula.

- 8. Einschichtige Keimblase. Planula (im engeren Sinne).

- 9. Gastrula - Gastraea.

Ec. Exoderm - Hautblatt.

En. Entoderm - Darmdrüsenblatt.

Lh. Leibeshöhle. Dh. Darmhöhle. 


\section{$2 \mathrm{BHL}$ Biodiversity Heritage Library}

Rolph, W H . 1874. "Ueber die genealogischen Systeme Haeckels, besonders die sog. Gastraeatheorie." Berliner entomologische Zeitschrift / herausgegeben von dem Entomologischen Vereine in Berlin 18(3प4)433-441. https://doi.org/10.1002/mmnd.18740180325.

View This Item Online: $\underline{\text { https://www.biodiversitylibrary.org/item/36410 }}$

DOI: https://doi.org/10.1002/mmnd.18740180325

Permalink: https://www.biodiversitylibrary.org/partpdf/210022

\section{Holding Institution}

Smithsonian Libraries

\section{Sponsored by}

Smithsonian

\section{Copyright \& Reuse}

Copyright Status: Public domain. The BHL considers that this work is no longer under copyright protection.

This document was created from content at the Biodiversity Heritage Library, the world's largest open access digital library for biodiversity literature and archives. Visit BHL at https://www.biodiversitylibrary.org. 